\title{
TRIS: THERMAL REMOTE IDENTIFICATION SYSTEM OF FEVERISH PEOPLE
}

Diogo A. Martins, Pedro P. V. Tecchio, João V. Torres Borges, Nelson A. Ferreira Neto, Ana C. B. de Jesus, Jéssica L. Motta, Aziel M. de Freitas Júnior, Mateus S. de Cerqueira, Tiago P. de Souza

Robótica e Sistemas Autônomos, SENAI CIMATEC, Brazil

Abstract: The COVID-19 pandemic has pressed for many technological fronts in the combat against the corona virus spreading. Among those fronts, the TRIS project raises up as a virus propagation control tool by assisting in the process of detecting feverish people, enabling the conduct of a medical thermal screening procedure. Among the functionalities proposed by TRIS, the essential modules are face detection and recognition, as well as, temperature estimation. In this paper, we point out the challenges and problems faced during its development and provide a comparison with other thermal systems used for face temperature estimation.

Keywords: COVID-19; artificial intelligence; face detection; temperature estimation, thermal screening

\section{TRIS: SISTEMA TÉRMICO REMOTO DE IDENTIFICAÇÃO DE PESSOAS FEBRIS}

Resumo: A pandemia de COVID-19 instigou muitas frentes tecnológicas no combate à propagação do corona vírus. Dentre essas frentes, o projeto TRIS surge como uma ferramenta de controle de propagação do vírus ao auxiliar no processo de detecção de pessoas febris, possibilitando a realização do procedimento médico de triagem térmica. Dentre as funcionalidades propostas pelo TRIS, os módulos essenciais são detecção e reconhecimento de faces, além de estimativa de temperatura. Neste artigo, apontamos os desafios e problemas enfrentados durante seu desenvolvimento e fazemos uma comparação com outros sistemas térmicos usados para estimativa de temperatura facial.

Palavras-chave: COVID-19; inteligência artificial; detecção de faces; estimação de temperatura, triagem por temperatura 


\section{INTRODUCTION}

Infectious diseases are a constant challenge for health and science. The emblematic case in the $21^{\text {st }}$ century is the new coronavirus that causes the COVID-19 disease, which despite the fact that science has already advanced and circumvented situations such as Ebola and the H1N1 influenza virus, faces endless difficulties about the capability of the new virus mutation. $18,902,735$ cases of COVID-19 and 709,511 deaths have been confirmed by World Health Organization, until August 7, 2020 [1] and this numbers tend to increase if there are no efforts to prevent coronavirus proliferation until a vaccine release.

In order to implement new strategies for the coronavirus prevention, a system named TRIS - Thermal Remote Identification System was developed. TRIS is an artificial intelligence based tool for remote temperature measurement that uses facial recognition on surveillance cameras and thermal cameras over crowded or intense pedestrian traffic places with the purpose of reducing infections caused by coronavirus.

This paper will describe and explain the challenges of developing technology to prevent coronavirus at times of pandemic. First of all, it will be presented the methodology, techniques and principles used as face detection, facial recognition, how the temperature is estimated, how the system interface works and data capture. Then the third section reports the results and analysis achieved with images and graphics.

\section{METHODOLOGY}

\subsection{Face Detection}

The face detection module determines the location, size and estimated pose of a face in a digital image. Face detection is carried out with RetinaFace, a robust singlestage face detector, which performs pixel-wise face localization on various scales of faces by taking advantages of joint extra-supervised and self-supervised multi-task learning [2]. RetinaFace outperforms the state-of-the-art average precision on WIDER FACE benchmark [3] and provides implementation examples in its repository.

This module was implemented in TensorFlow 2.0+ with ResNet50 as the convolutional backbone architecture used for feature extraction. The neural network model was trained and validated with WIDER FACE dataset, achieving an average precision of $83.55 \%$ on the hard test set. Within the TRIS system, the face detection module receives images from cameras as input, executes the inference and returns the set of bounding boxes corresponding to the faces found.

The Face Detection and subsequent recognition are done in images acquired by a Logitech C270 camera, Figure 1a, and by a FLIR ADK, Figure 1b. The Logitech C270 has a resolution of $1280 \times 720$, 30 FPS (Frames Per Second) and a FOV (Field of View) of $60^{\circ}$. The FLIR ADK has a resolution of $640 \times 512$, being able to operate with 30 or 60 FPS and a FOV of $50^{\circ}$. 
Figure 1: TRIS cameras

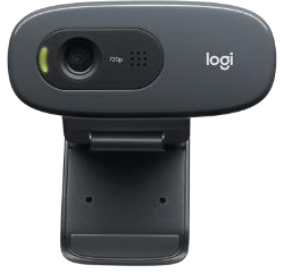

Figure 1a: Logitech c270

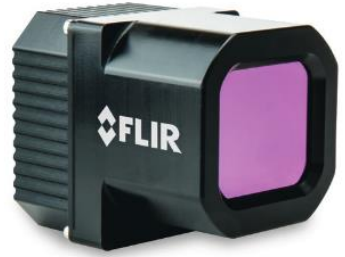

Figure 1b: FLIR ADK

\subsection{Facial Recognition}

The system is also equipped with facial recognition capabilities, enabling the tracking of individuals of interest (the feverish ones). This feature is based on the face_recognition framework [4]. It works by processing the RGB captured frames as an input, then it proceeds to assign face signatures to each detected face. The framework uses a deep learning trained model, that ran through approximately 3 million faces of more than 7000 different individuals, in order to give the algorithm an ability to recognize where are the most distinctive features in a face, which then enables recognition [5].

As a next step of this project, all successfully detected faces on each camera captured frame will have their signatures compared against database stored face signatures and the registered individuals whose temperature are beyond the safe threshold will be contacted and informed of the fact.

\subsection{Temperature Estimation}

In order to estimate human face temperature from infrared radiometric images, one needs to first convert these images which contains values proportional to the amount of infrared radiation generated by scene objects to temperature values. Some cameras already provide thermal images, which are the ones with temperature values for each pixel, others do not. In this work a camera that did not have this feature FLIR ADK was used, Figure $1 \mathrm{~b}$. It has a resolution of $640 \times 512,30$ or 60 FPS and a FOV of $50^{\circ}$. Its thermal sensitivity is $50 \mathrm{mK}$ or less.

Because of that, a simple experiment of heating a body of water was developed to obtain the camera calibration curve that relates radiance to temperature values. The water temperature was measured using a Minipa ET-2082B with the provided thermocouple. This equipment has a $1^{\circ} \mathrm{C}$ resolution over the range of measured temperatures, which was around 10 to $100{ }^{\circ} \mathrm{C}$. Later on, this calibration curve was used to estimate the temperature values throughout this work.

After temperature conversion, one needs to estimate the face temperature from the bounding boxes of each image given by the face detection and recognition algorithm. We propose a straightforward statistical approach of only using data from the $75^{\text {th }}$ to $95^{\text {th }}$ percentiles which are then averaged to obtain an estimate. This estimate is later compared with a given temperature threshold to decide if the person has a fever or not. Another approach, would be to utilize a calibrated "black body" as a thermal reference on the scene captured by the thermal camera. 


\subsection{Dataset Capture}

While still under study and implementation, the dataset capture feature will allow one to record a set of RGB and infrared radiance video files or still frames. It is already possible to store video files from both cameras using image manipulation and video compression techniques.

While most color cameras and video files utilize three layers of unsigned 8 bits values per pixel, raw radiance images are received from the infrared thermal camera as a single layer of unsigned 16 bits values per pixel, as seen on Figure 2 . Therefore, in order to utilize standard video compression encoders to store uncompressed radiance images, the single layer of 16 bits values per pixel were segregated in two layers of 8 bits values per pixel by utilization of bit-shifting. These two layers were then coupled with another zeroed layer to form a standard RGB image, which was then compressed as usual.

Figure 2. Thermal image example in raw 16 bits
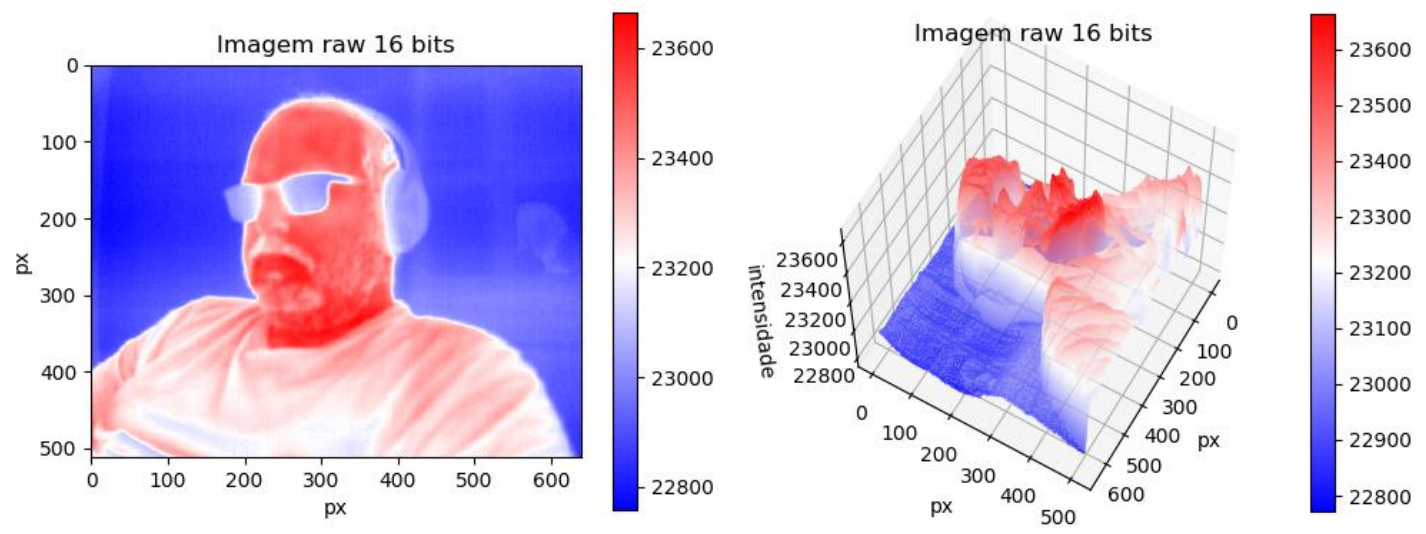

\section{RESULTS AND DISCUSSION}

\subsection{Operation of the Graphical User Interface - GUI}

The TRIS GUI allows a verified user control of the system operation. User verification is done via login and password credentials check. By command of the user, the system also shows, in real time, both RGB and infrared camera streams with or without bounding boxes marking all detected faces and their estimated temperatures. In Figure 3 is shown a picture of the graphical user interface. 
Figure 3: TRIS interface.

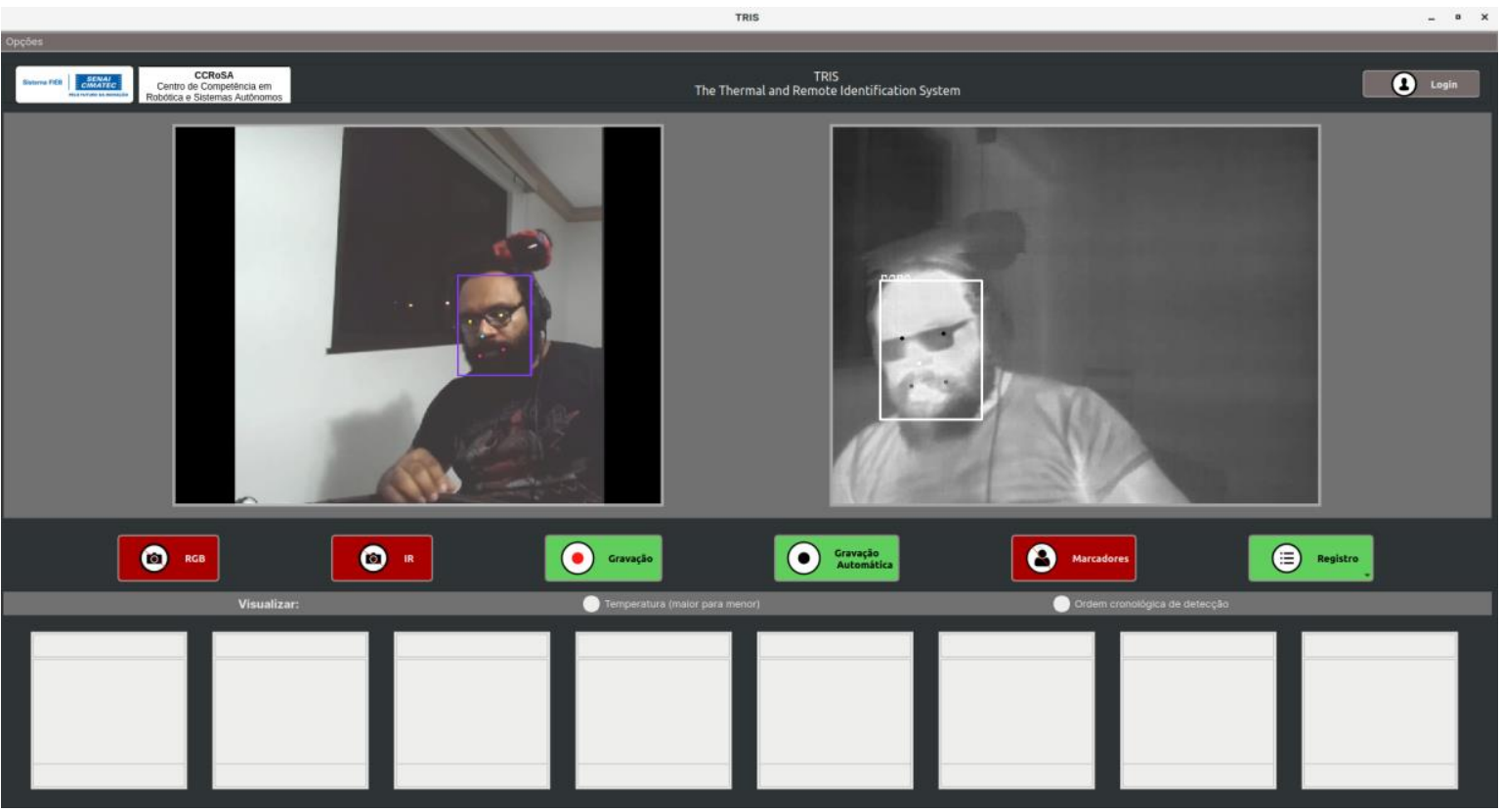

The system may show in the bottom half of the interface the faces of people classified with a feverish state. The user is able to choose the display order by decreasing temperature values or by increasing detection time. If a face can be matched to a person in the system database, his or her identity will is shown along with his or her face and temperature. If no match occurs, only the detected face and temperature is shown. All the above information will be kept by the system so that it can be consulted at any time.

\subsection{Inference Optimization}

In order to meet real-time operating requirements, the optimization of the neural network graph was performed through the TensorFlow integration with NVIDIA TensorRT (TF-TRT). Two optimized models were generated, one with the neural network weights represented in 32-bit floating point values (FP32) and another with half-precision float point values (FP16). The performance of the system with TensorFlow and TF-TRT optimized models can be seen in Tables 1 and 2.

Table 1. System performance values: Development environment Ubuntu 18.04, AMD Ryzen 7 1800x, 16GB DDR4, NVIDIA RTX 2070 Super.

\begin{tabular}{|l|c|c|c|}
\hline & $\begin{array}{c}\text { TensorFlow } \\
\text { (FPS) }\end{array}$ & $\begin{array}{c}\text { TF-TRT - FP32 } \\
\text { (FPS) }\end{array}$ & $\begin{array}{c}\text { TF-TRT - FP16 } \\
\text { (FPS) }\end{array}$ \\
\hline Minimum & 2.57 & 30.96 & 31.09 \\
\hline Mean & 3.15 & 34.38 & 34.56 \\
\hline Standard deviation & 0.17 & 1.77 & 1.76 \\
\hline
\end{tabular}




\begin{tabular}{|l|c|c|c|}
\hline Maximum & 3.67 & 39.06 & 39.72 \\
\hline \multicolumn{2}{|c|}{$\begin{array}{r}\text { Table 2. System performance values: Test environment Ubuntu } \\
\text { 8GB DDR4, NVIDIA GTX 1650. } \\
\text { TensorFlow } \\
\text { (FPS) }\end{array}$} & $\begin{array}{r}\text { TF-TRT - FP32 } \\
\text { (FPS) }\end{array}$ & $\begin{array}{c}\text { TF-TRT - FP16 } \\
\text { (FPS) }\end{array}$ \\
\hline Minimum & 3.39 & 16.33 & 20.45 \\
\hline Mean & 4.17 & 23.45 & 23.72 \\
\hline Standard deviation & 0.12 & 1.56 & 1.16 \\
\hline Maximum & 4.44 & 26.24 & 27.09 \\
\hline
\end{tabular}

\subsection{Thermal Camera Curve Calibration Experiment}

Both heating water experiments results are displayed in Figures 4 and 5 . As can be seen in these figures, a simple linear function was enough to provide a good enough fitting model with R-squared values greater than 0.99 in both cases. But, although both experiments can be fitted to a linear model, the resulting linear functions are distinct in both slope and intercept values. It is relevant to point that both experiments were done using the same materials, but in different days.

Figure 4. First thermal camera curve calibration experiment

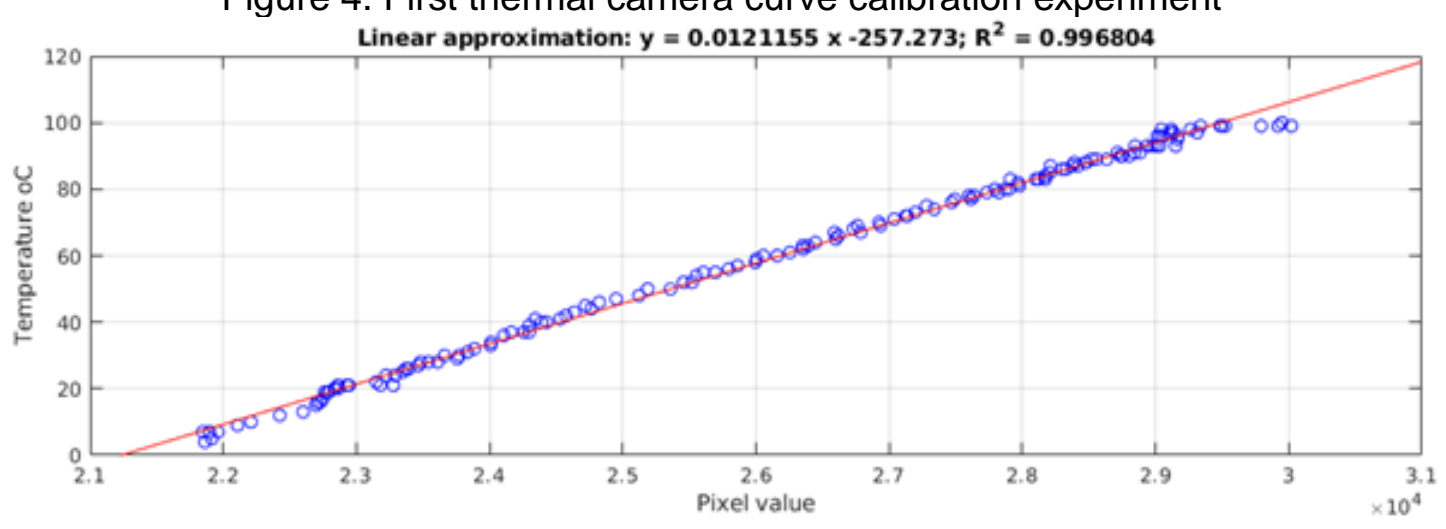

Figure 5. Second thermal camera curve calibration experiment

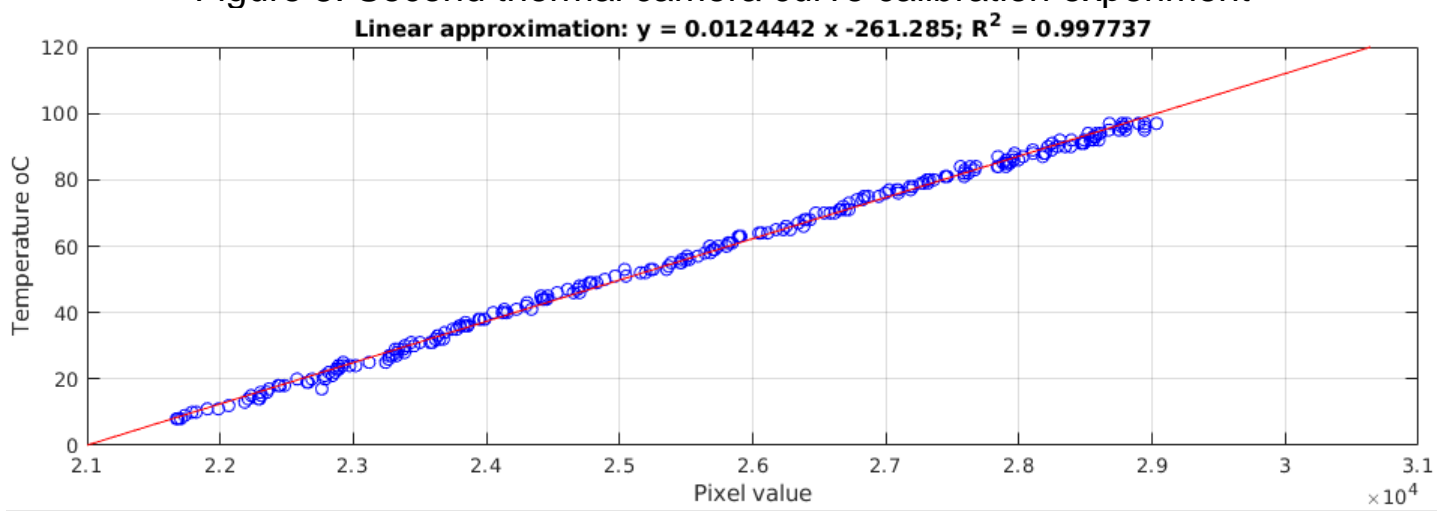


These results seem to indicate that the calibration curve may be approached as a linear function by themselves, but directly applying these linear models during further development of this project did no provide consistent results. Temperature differences from ground truth to estimated values of up to $5^{\circ} \mathrm{C}$ were found during adverse ambient temperature and sunlight illumination. Thus, TRIS is a solution to be tested under controlled environmental conditions, with suitable distances between cameras and pedestrian and far from sunlight exposition. These results seem to indicate that a more robust non-linear model which takes in consideration other factors besides object instantaneous temperature and radiance values is required for greater accuracy and reliability.

Figure 6. Infrared camera behavior of static scene over time (frames)

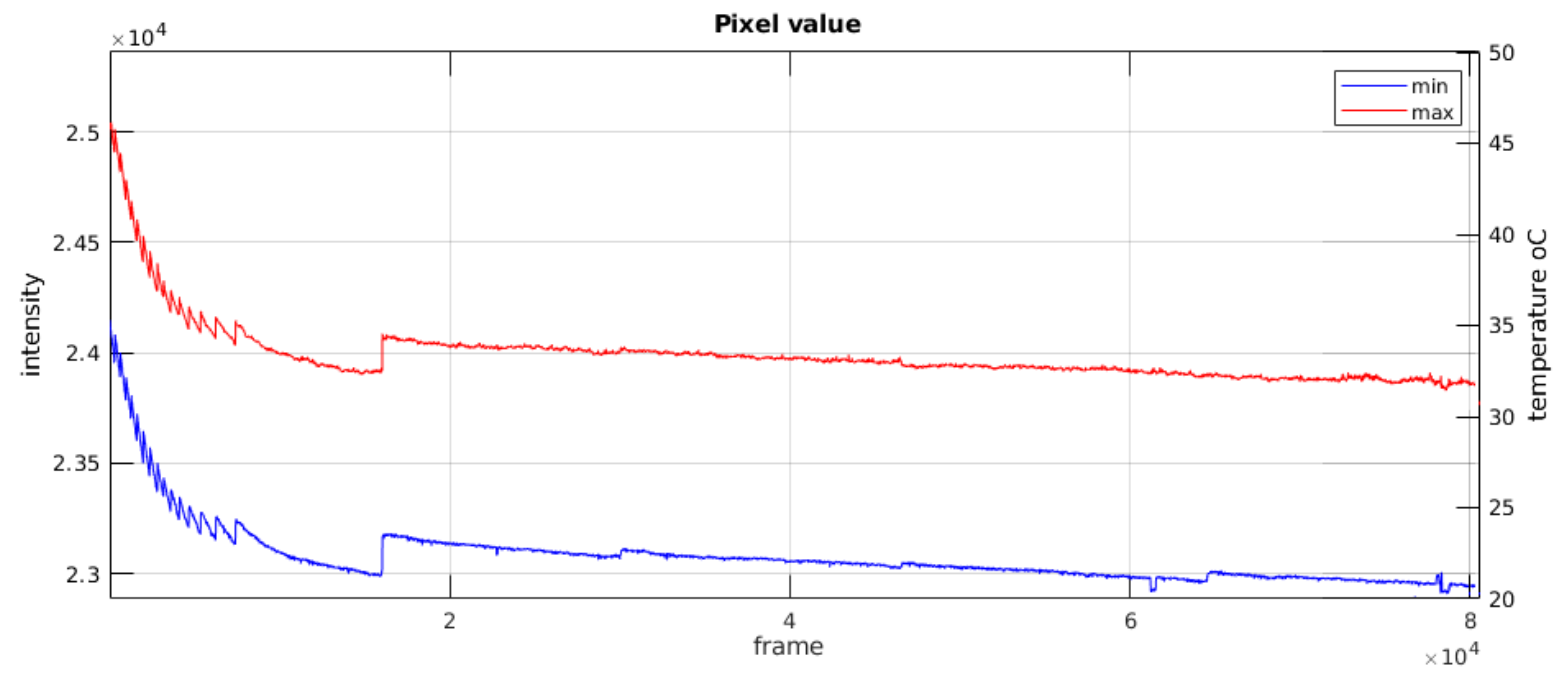

Moreover, as can be seen from Figure 6, the existing infrared camera has pixel intensity drift over different image frames taken from the same static scene. This behavior causes further issues in the temperature estimation process, it is not easily corrected and usually requires an improved hardware configuration.

\section{CONCLUSION}

TRIS utilizes state of the art techniques like RetinaFace neural network architecture for face detection and inference optimization with TensorRT. These combined with our temperature calibration experiments allows TRIS to provide its user with an estimated temperature for every detected person passing through its line of sight in real time.

While the current solution does not conform with the desired accuracy (about $\pm 0.3^{\circ} \mathrm{C}$ ), it is important to notice that commercially available state of the art thermographic cameras have accuracy around $\pm 2{ }^{\circ} \mathrm{C}[6,7]$. Moreover, this accuracy happens while taking account of more robust physical models and improved calibration methods. Existing commercial solutions for human fever screening make use of the same calibration methods in a much more restrained temperature range centered around normal human body temperature $[8,9]$. These systems can also use external temperature reference sources known as blackbodies in order to further increase their accuracy [9]. The results shown in this work do not use these methods yet.

More accurate temperature estimations will be achieved during the current system development using a more reliable calibration procedure, by taking account of 
emissivity, reflectivity and transmissivity constraints, as well as, environment variations and by making use of external temperature references and a more appropriate thermal camera. TRIS is expected to be employed as sensory tool to allow for control the spread of diseases which cause feverish states, specially the coronavirus. It is currently being tested at SENAI CIMATEC in one of its laboratories in order to verify its performance and reliability. These tests will provide data for further development.

\section{Acknowledgments}

The authors would like to thank SENAI CIMATEC for its support on materials and funding for this research.

\section{REFERENCES}

1 BASTOS, L., 2020. OPAS/OMS Brasil - Folha Informativa - COVID-19 (Doença Causada Pelo Novo Coronavírus) / OPAS/OMS. [online] Pan American Health Organization / World Health Organization. Available at:

$<$ https://www.paho.org/bra/index.phpoption=com_content\&view=article\&id=6101:covi d19\&Itemid=875>. Accessed on: 10 Aug. 2020.

2 DENG, Jiankang et al. RetinaFace: Single-Shot Multi-Level Face Localization in the Wild. In: Proceedings of the IEEE/CVF Conference on Computer Vision and Pattern Recognition. 2020. p. 5203-5212.

3 YANG, S. et al. Wider face: $A$ face detection benchmark. IEEE Conference on Computer Vision and Pattern Recognition (CVPR), 2016.

4 GEITGEY, Adam. Face recognition. Available at: <https://github.com/ageitgey/face_recognition>. Accessed on: 10 Aug. 2020.

${ }^{5}$ KING, Davis. Dlib Models. Available at: <https://github.com/davisking/dlib-models>. Accessed on: 10 Aug. 2020.

${ }^{6}$ FLIR® Systems, Inc. FLIR A400/A700 Thermal Cameras with Smart Sensor Configuration. Available at: <https://www.flir.com/products/a400-a700-smartsensor/?model=85900-0000-T300293> Accessed: 13 Sep. 2020.

${ }^{7}$ Fluke Corporation. Fluke RSE600 mounted infrared camera. Available at: < https://www.fluke.com/en/product/thermal-cameras/rse600> Accessed: 13 Sep. 2020.

${ }^{8}$ FLIR® Systems, Inc. FLIR A700 EST Thermal Screening Solutions. Available at: < https://www.flir.com/products/flir-a700-est/?model=85902-0101> Accessed: 13 Sep. 2020.

${ }^{9}$ Seek Thermal. Seek Scan ${ }^{\mathrm{TM}}$. Available at: < https://www.thermal.com/seekscan.html> Accessed: 13 Sep. 2020. 